\title{
Supporting Statistics in the Workplace: Experiences with two Hospitals
}

\author{
M. Y. MORTLOCK† \\ m.mortlock@fsc.qut.edu.au \\ School of Mathematical Sciences, Queensland University of Technology, Australia \\ N. SPENCER \\ nancy.spencer@treasury.qld.gov.au \\ Office of Economic and Statistical Research, Queensland Treasury, Australia \\ K. MENGERSEN \\ kerrie.mengersen@newcastle.edu.au \\ School of Mathematical and Physical Sciences, University of Newcastle, Australia
}

\begin{abstract}
This paper provides some reflections on the promotion of lifelong learning in statistics in the workplace. The initiative from which the reflections are drawn is a collaboration between a university and two public hospitals, of which one of the stated aims is to develop statistical skills among the hospitals' researchers. This is realized in the provision of 'biostatistical clinics' in which workplace teaching and learning of statistics takes place in one-on-one or small group situations. The central issue that is identified is the need to accommodate diversity: in backgrounds, motivations and learning needs of workplace learners (in this case medical researchers), in the workplace environments themselves and in the projects encountered. Operational issues for the statistician in providing such training are addressed. These considerations may reflect the experiences of the wider community of statisticians involved in service provision within a larger organization.
\end{abstract}

Keywords: adult education, biostatistics, statistical consulting, work-based learning

\section{Introduction}

Adult learning in the workplace is a valued and integral part of many institutions. This paper reflects on the operational and statistical issues arising from conducting a work-based biostatistics clinic for staff at two public hospitals in Brisbane, Queensland. The statistical support is provided as part of a wider Strategic Partnerships with Industry Research and Training (SPIRT) scheme. The SPIRT grant scheme supports a cooperative reliance between higher education institutions, industry, commerce and the public sector. In this case, Queensland University of Technology (QUT) School of Mathematical Sciences cooperates with two public hospitals in Brisbane

$\dagger$ Requests for reprints should be sent to M. Y. Mortlock, School of Mathematical Sciences, Queensland University of Technology, Australia. 
(The Princess Alexandra Hospital and the Prince Charles Hospital). Both these hospitals benefit from a weekly biostatistics clinic available to staff at the hospitals. In hospitals, where evidence based practice is practised, statistical support provided by an in-house statistical clinic aims to enhance research outcomes.

Dunkin [2] discusses the relationship between work and learning and highlights the changing nature of the employer expectations. Employees are expected to be effective participators who are able to communicate, adapt to new technologies, work in a team, make judgements, and take initiative. In the process of statistical consultation, such outcomes are encouraged as specific help is provided in a work-embedded problem, where knowledge and practice are integrated. In fact specialization and flexibility need to coexist and this requires the capacity to continually learn to both increase one's depth of knowledge and to have vision for its application and relevance in an ever-changing technological environment. Critical constructivist teaching [8] enables the learner to be actively and critically involved in their own learning, and the consultant (or teacher) to facilitate the process of empowering the learner. In the case of consulting, this is a useful model as it recognizes the interdependence of the teaching/learning experience. Within this initiative we provide work-based statistical support. Medical scientists at two hospitals have access to weekly statistical support with a relevant and problem based focus.

Ritter [6] describes a range of competencies required by statisticians. The job responsibilities of a statistician include the 'statistical' competencies on specific theories or methods and, the 'technical', referring to computer related and mathematical, and the 'non-statistical', an ability to participate and work in teams, to make presentations and to have a rapport or personal style that allows an effective educative and consulting role. Thus we require, not only good core statistical knowledge but also good 'people' skills.

Consulting in the workplace can be successful for both the learner and the statistician if there is clear open communication, and realistic expectations. Perhaps the most important issue identified through our experience in this project is the need to recognize and accommodate diversity. This diversity occurs because of different workplaces, different projects, different backgrounds and abilities of learners and teachers, and different motivations for learning. This paper discusses how the clinic is conducted with some notes on feedback. Following on are sections on project diversity, diversity of learners, working in the work based environment (in the hospitals) and some personal and professional outcomes reflected from an 18-month pe- 
riod of operation. The discussion uses a framework of the dimensions of problem-based learning identified by Reeves [5].

\section{The Biostatistics Clinic}

The statistical clinics operate once a week at each of two hospitals. The booking is via e-mail, and a typical consultation lasts one hour and is offered to individual practitioners and researchers. Typically, up to five consultations are provided in a day at the hospital, with flexibility to meet at other times at the university as required. The clinic operates throughout the year so users can organize a single visit or a series of visits to meet their needs.

Typically, the initial consultation begins with the statistician inviting a description of the research problem, with emphasis on refining the questions to be asked. E-mail contact prior to the meeting can be efficient in providing research plans, data descriptions, and potential statistical issues ahead of the consultation. By considering the data type and expected differences or expected associations an appropriate statistical technique or alternatives are recommended. Researchers are encouraged to check their data, list and describe their variables and document their data coding, refine their research questions and to undertake exploratory data analysis. Interpretation and further analysis is suggested as appropriate. Assistance is provided from advice on experimental design, data collection, planning of pilot studies, through to publication.

Occasionally e-mail is sent to clients, requesting a brief summary of the help that was given via the clinic, and inviting some comments on the service. This allows us to try to improve the service. The following emailed comments came from three researchers:

- "Feedback: Great - ...the statistics were confirmed by you and this helped my confidence that I was on the right tract and understood the outcomes."

- “.... I was appreciative of being able to meet with someone on campus and secondly appreciated the willingness to collaborate with another university"

- "Feedback: Very helpful in determining the type of method used in the project."

In addition, the project keeps a file of contacts and a brief description of the type of consultation, papers presented, and papers published. 


\section{Diversity of Projects}

A range of statistical assistance is provided. Some projects are in their infancy and discussions focus on the research hypothesis, and consolidate an idea into a pilot study. Other projects are interested in sample size and study design, in particular, in writing for research funding. Other projects require advice on data collection and handling. Issues include the survey instruments; for example, in quality of life questionnaires, the way to code data. Projects seek advice on appropriate designs and help with randomization, or checking the data for conforming to the correct assumptions for the analysis. One factorial experiment looked at three factors of interest on resistances of airflow in various commercial types of tubing. Other experiments may involve repeated measures due to repeated times of measurement or in one case, the successive increase in dose of a drug that was added by increments. Assistance has been given in software use; for example, with exploratory data analysis and logistic regression. Other projects seek assistance in writing statistical methodology for publication.

\section{Diversity of Participants}

One of the best motivations for understanding a topic is when one has to use it. Medical courses differ in the amount of biostatistics provided. Sahai [7] discusses the reasons why biostatistics may not be given the same enthusiasm as other aspects of the medical training. Statistical literacy is considered a necessity for efficient understanding and practising of science [1]. In statistical consulting, which is a work based form of adult education, it is important to be aware of the individual learners different backgrounds. This comprises differences in statistical education and other training, stages in their research careers and different interests in statistics and motivations in their work. The diversity is to be expected when the consultation is to individuals at different stages in their research careers, and with different interests in statistics and motivations in their work.

Reeves [5] discussed several pedagogical dimensions that emerge in a problem-based learning environment. He describes each of seven dimensions on a continuum. These are presented below, and these dimensions are applicable to the variety of learners visiting the statistical clinic. Some are statistical novices, early in their research careers, and this contrasts to others who are very experienced and have excellent statistical skills.

Goal orientation: From Specific focus to General focus

Goal orientation ranges from getting the job done as easily as possible 
through to wanting to develop their own deeper appreciation of statistics. Some learners prefer to hand over the data and concentrate their efforts in other areas. Others wish to progress to a deeper understanding of statistics.

\section{Pedagogical approach: From Instructivist to Constructivist}

The latter type fosters critical reflection in adulthood and combines research-based knowledge and experienced-based knowledge to contribute to excellence in adult teaching [3]. To understand the scholarship of teaching [3] we need to reflect on this and on our practise on our demonstrated knowledge of teaching.

Instruction/supervision: From Didactic to Facilitative

From their studies or interaction with supervisors some researchers may be used to particular approaches to learning and the encouragement of self-development through the critical constructivist model may not be welcomed. The statistician must assist in the facilitation of learning applied statistics but start at the point at which the student is currently standing. Nature of activities: From Replication to Generation The consulting activities emphasize examining the learners' own data. However, in the early stages of planning we encourage the search for similar data elsewhere to gain an appreciation of designs, hypotheses, implementation and variability. Both ends of this spectrum are integral to a statistical appreciation of data, its understanding and summarizing how it answers the questions in our research problem.

Source of motivation: From Extrinsic to Intrinsic

In some cases the clients are expert experimentalists while other learners need a lot of help with appraisal of design and evaluation of appropriate and/or alternate analysis. The motivation for the learning is to develop statistical skills for their particular experiment or study.

Experimental validity: From Abstract to Concrete

In most cases the projects represent a work-embedded concrete problem to solve. In some cases the objectives of a study may not clear, and in the process of consulting we work together to define these more clearly. In some cases a concrete starting point may lead to more abstract research questions and develop into kernels of an idea for a new experiment.

Cooperative learning: From Proscribed to Integral

This dimension considers whether the learning is integral to achievement. In most cases research results in outcomes such as abstracts, papers, or presentations and there is tangible use for the statistical learning experience. The working through data to achieve outcomes, such as a thesis, a journal article or a presentation is usually very motivating. In some instances the time frame may be very short, due to tight deadlines or due to 
the learner not realizing how much work is involved in a data preparation, data summary and analysis.

\section{Working in the Hospital}

There are ranges of specific issues that arise for a statistician in the hospital [4]. Some are very positive and include the excitement of dealing with primary data and contact with the collector of the data. Fostering inhouse expertise improves research teams' confidence and use of statistics, which allows a more efficient research process, from project formulation through to publication. Statistical consulting in a hospital is a rewarding experience as there is a demand for the service, and the regular clinics allow the statistician to become an integral part of an office. For the educator, it provides motivation as one is continually exposed to new projects or work challenges on an immediate basis. There is the potential for the statistician to enhance their ability to dissect and evaluate a problem with the frequent attention to many new projects to appraise.

Some other issues make the operating of a clinic more difficult at the work based site compared to the home institution. These issues include dealing with people, some who may have unrealistic expectations. Dealing with researchers who wish to test their data for associations when the data are not suitable for this purpose requires diplomacy and an ability to explain both the reasons for statistical rigor, and the need to plan the analysis at the initial stage. Most data sets need a lot of cleaning and tidying, even for exploratory data analysis.

There is a lack of contact with fellow statisticians. Some researchers are about to leave the hospital or even the country and have a limited time frame for completing their analysis. It is harder to get computing and IT support exactly when you need it due to using a lap-top from the home institution, rather than part of hospital system. As an adjunct to a project, the statistician usually has little control on the time frame for analysis and publication. Often one is required to have access to several computer networks, which is time consuming. The statistician needs to be comfortable with multiple office locations and maintain flexibility and remain well organized despite this. The role involves added travel time and organizational time compared to working from a single location.

Working away from the main department, like any 'field work', requires the statistician to be well organized. The laptop, mobile phone and e-mail allow one to operate at different sites on different days with the 'mobile office'. There is some isolation from other departmental colleagues, and it 
isn't possible to attend all meetings and seminars at the two hospitals and the university.

\section{Personal and Professional Outcomes}

The statistician also needs to have some outcomes apart from the satisfaction of operating the clinics. The workplace environment has a different structure to the university department. There is no 'endpoint' in terms of assessment compared to that on a more formal course whereby the teacher can obtain feedback. Other types of success can be measured by whether there are constant referrals, and whether the learner is able to do the task and has gained understanding. The output of papers and presentations with co-authorship, and acknowledgments can be one measure, although there is often a considerable lead-time for these.

The project also conducts quarterly workshops, which focus on particular concerns and applied medical statistical problems. Statistical issues are highlighted, and expanded on for the benefit of the whole group. This allows contact between fellow statisticians and provides a forum to communicate with statisticians from QUT and researchers from the two hospitals. At one hospital ad hoc seminars were offered to provide further support to groups requiring some overview of particular statistical methods. This allows a group of up to twenty people to have a focused statistical topic to be presented in-house. Future seminars will include a data set provided by the group, which will subsequently be used to explain exploratory data analysis through to survival analysis. A focus on outcomes (such as case studies from the clinics) is important for professional self-development and helps to maintain motivation. Statisticians needs to feel confident in their ability to deal with the diverse requests. It is useful to also involve young statistical graduates from time to time in the consulting, enabling them to develop some practical experience. Allowing graduate statisticians to participate and to take on some parts of the analysis can broaden their experience and provide useful backup for a busy clinic.

\section{Discussion}

The clinics are well supported, as they are fully booked up most weeks. The SPIRT initiative has strengthened the statistical support for a wide range of projects. The clinics on site at the hospitals make statistical support very accessible to busy medical staff, and follow-up e-mail with comments and analysis makes the process very effective. 
This collaborative project between hospitals and the QUT School of Mathematics provides benefits for both medical scientists and statisticians. The project provided support to two hospitals, and this paper attempts to discuss the diversity of issues that can arise for the practising statistician. Many of the work based practise experiences would apply to other 'on-site' statisticians. There is a need to maintain a balance among the many projects, some established and the several new ones that continually present to the clinic, and the multiple roles of educator, and presentation of statistical advice. There is also a need to focus on outcomes for the project including papers, workshops, case studies and statistical networking and progress in statistical understanding. There are positive outcomes for the collaborators. On the one hand the hospital researchers have gained an increase in statistical skills, and can easily access a statistician on site. The statistician gains access to new and exciting data sets and is be able to develop novel approaches and write-up case studies as a technical, applied reference for the medical scientist. The synergies of working closely provide outcomes for research for all participants.

\section{Acknowledgments}

This project was supported by an ARC SPIRT Grant number C10024120.

\section{References}

1. P.B. Cerrito. Teaching statistical literacy. College Teaching, 47(1): 9-13, 1999.

2. R. Dunkin. The integration of work and learning: the learner as a person. Effective Teaching and Learning Conference, QUT, Brisbane, 12 November, 2001.

3. C. Kreber and P.A. Cranton. Exploring the scholarship of teaching. The Journal of Higher Education, 71(4):476-495, 2000.

4. M.Y. Mortlock. Providing biostatistical support in work-based environments in health: reflections on teaching and learning issues. Effective Teaching and Learning Conference, QUT, Brisbane, 12 November, 2001. (http://www.talss.qut.edu.au/etl/presentation/presentation_outlinesWed.htm)

5. T.C. Reeves and J.M. Laffey. Design, assessment and evaluation of a problem-based learning environment in undergraduate engineering. Higher Education Research and Development, 18(2):219-232, 1999.

6. M.A. Ritter, R.R. Starbuck and R.V. Hogg. Advice from prospective employers on training BS Statisticians. The American Statistician, 55:(1):14-18, 2001.

7. H. Sahai and M.M. Ojeda. Problems and challenges of teaching biostatistics to medical students and professionals. Medical Teacher, 21(3):286-288, 1999.

8. M. Watts and Z. Jofili. Towards critical constructivist teaching. International Journal of Scientific Education, 20(2):173-185, 1998. 


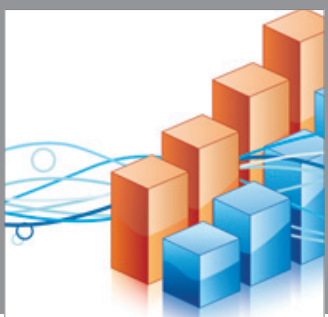

Advances in

Operations Research

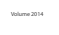

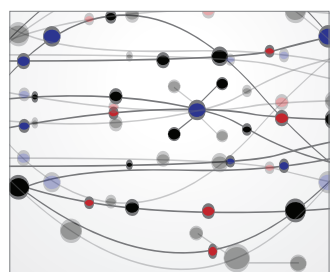

\section{The Scientific} World Journal
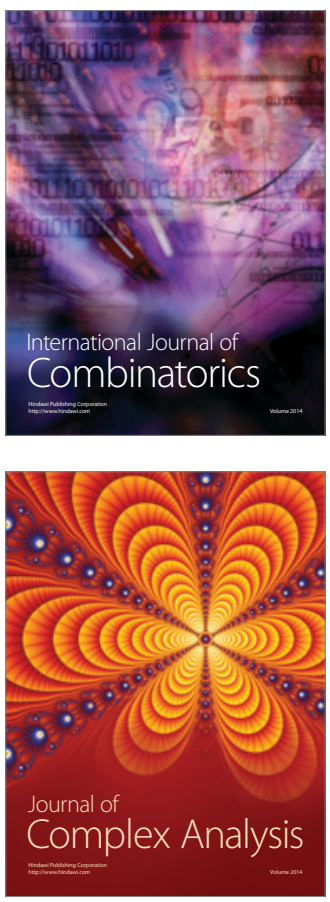

International Journal of

Mathematics and

Mathematical

Sciences
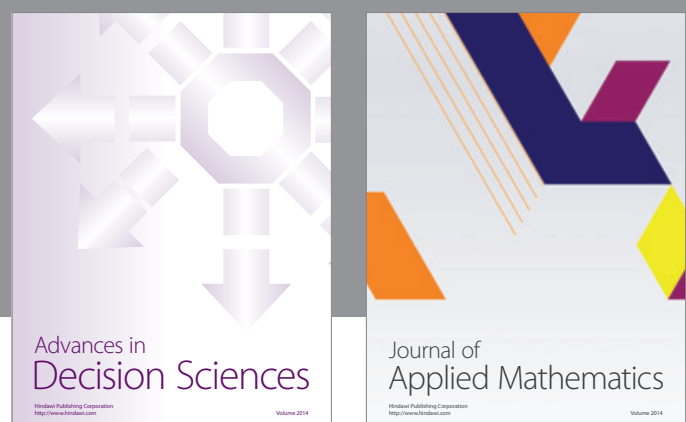

Journal of

Applied Mathematics
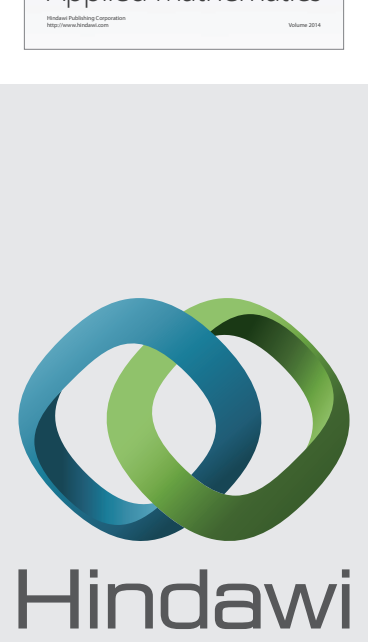

Submit your manuscripts at http://www.hindawi.com
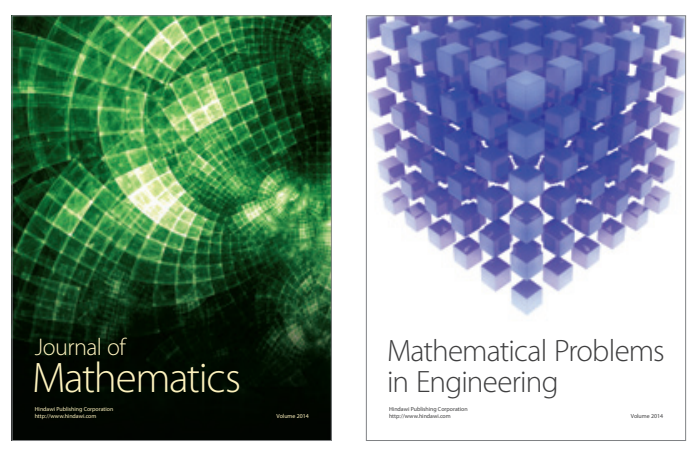

Mathematical Problems in Engineering
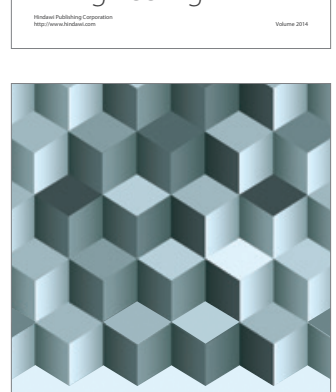

Journal of

Function Spaces
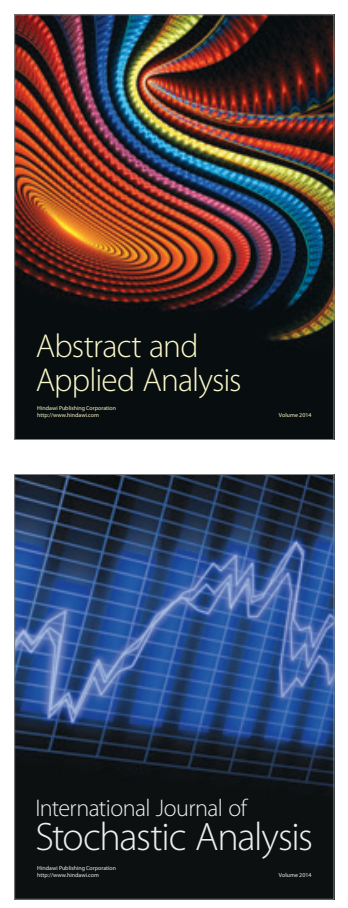

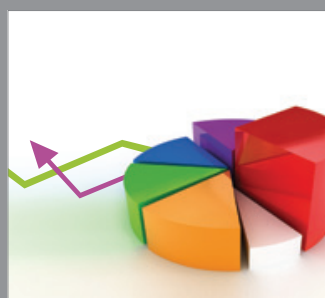

ournal of

Probability and Statistics

Promensencen
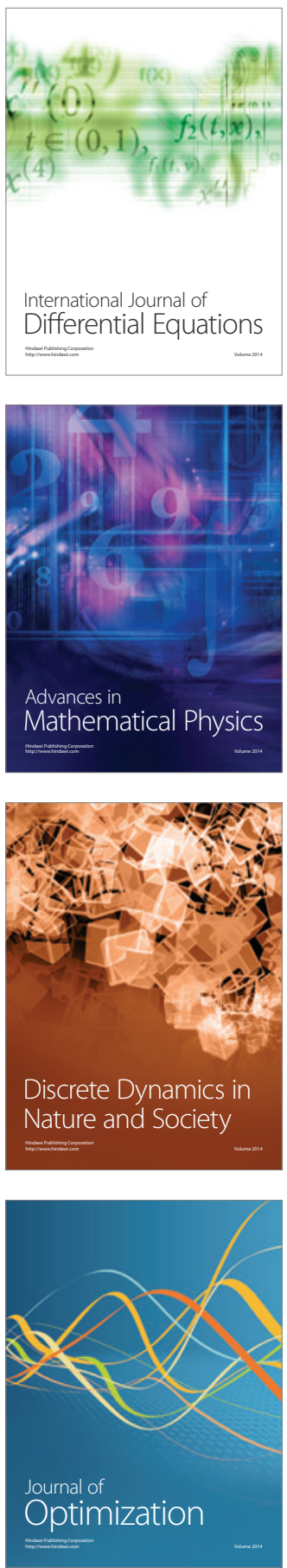